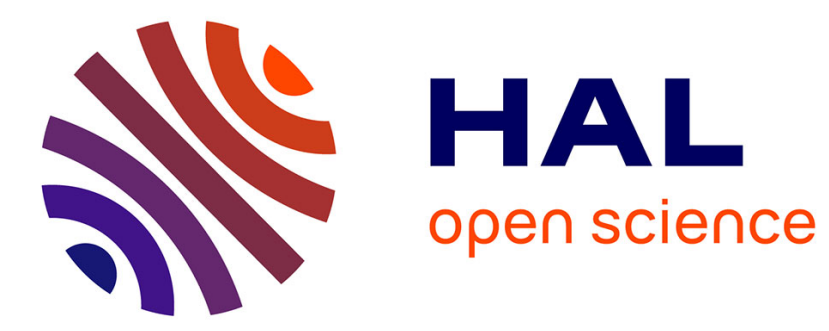

\title{
Doppler-free magnetic optical activity
}

\author{
S. Giraud-Cotton, V.P. Kaftandjian, B. Talin
}

\section{To cite this version:}

S. Giraud-Cotton, V.P. Kaftandjian, B. Talin. Doppler-free magnetic optical activity. Journal de Physique Lettres, 1980, 41 (24), pp.591-596. 10.1051/jphyslet:019800041024059100 . jpa-00231854

\section{HAL Id: jpa-00231854 https://hal.science/jpa-00231854}

Submitted on 1 Jan 1980

HAL is a multi-disciplinary open access archive for the deposit and dissemination of scientific research documents, whether they are published or not. The documents may come from teaching and research institutions in France or abroad, or from public or private research centers.
L'archive ouverte pluridisciplinaire HAL, est destinée au dépôt et à la diffusion de documents scientifiques de niveau recherche, publiés ou non, émanant des établissements d'enseignement et de recherche français ou étrangers, des laboratoires publics ou privés. 


\title{
Doppler-free magnetic optical activity
}

\author{
S. Giraud-Cotton, V. P. Kaftandjian and B. Talin \\ Département des Interactions Moléculaires (*), Université de Provence, Centre de St-Jérôme, 13397 Marseille Cedex 4, France
}

(Reçule 17 juillet 1980, révisé le 1er octobre, accepté le 20 octobre 1980)

\begin{abstract}
Résumé. - La théorie de l'activité optique magnétique sans effet Doppler d'une raie d'absorption isolée est présentée. La transmission d'un rayonnement laser balayable polarisé rectilignement à travers un milieu gazeux dilué est étudiée en présence d'un second laser saturant se propageant en sens inverse et d'un champ magnétique longitudinal constant. Pour cela on calcule la susceptibilité non linéaire au troisième ordre pour un système à deux niveaux présentant un effet Zeeman normal, à $J$ quelconque.
\end{abstract}

\begin{abstract}
The theory of Doppler-free magnetic optical activity associated with a single absorption line is presented. The transmission of tunable laser light, linearly polarized, through a dilute gaseous medium along a steady magnetic field is studied in the presence of a second counterpropagating saturating laser. The third order non linear susceptibility is calculated for a two-level system exhibiting a normal Zeeman effect, with arbitrary $J$ values.
\end{abstract}

1. Introduction. - When a linearly polarized light beam propagates through an absorbing medium placed in a steady magnetic field, parallel to the direction of propagation of the light, it becomes elliptically polarized. The major axis of the ellipse is rotated by an angle $\theta$ from the initial direction of polarization (Faraday effect). The ellipticity $\Phi$ of the emerging light results from the circular dichroism and the angle $\theta$ from the circular birefringence induced by the magnetic field. Both phenomena which are functions of the frequency, $\omega$, are covered by the general name of " magnetic optical activity " (MOA); they are related to the electrical susceptibility tensor $\chi^{(\mathrm{e})}$ of the medium, through [1];

$$
\theta+i \Phi=\frac{2 \pi \omega l}{i c} \chi_{x y}^{(\mathbf{e})}
$$

where the $z$-axis is taken along the direction of the magnetic field and the direction of propagation of the light. The MOA is usually observed through the magnetic rotation spectra (MRS), i.e. the intensity of radiation transmitted through crossed polarizers [1]

$$
I=I_{0}\left(\operatorname{sh}^{2} \Phi+\sin ^{2} \theta\right) \exp \left[-\left(\alpha_{\mathrm{R}}+\alpha_{\mathrm{L}}\right) l / 2\right]
$$

$l$ is the sample length, $\alpha_{R, L}$ is the absorption coefficient

(*) Equipe de recherche associée au C.N.R.S., $\mathrm{n}^{\circ} 898$. for right and left circularly polarized wave, and $I_{0}$ is the reference intensity.

MRS have been investigated for several monoatomic vapours, and a number of diatomic gases and molecular complexes [2] in the visible and near infrared regions. They provide the same informations on molecular or atomic structures than classical absorption spectroscopy and, moreover, specific informations on very small magnetic moments, electronic states perturbations [3], bands heads of some vibrational spectra [4], coupling schemes and transition moments [5] of molecules.

In recent years the improvement in spectroscopic technique arising from tunable visible and near infrared lasers has been used to accurately determine the structure and shape of atomic [6] and molecular lines.

However, MOA has been little used quantitatively in these spectral regions, since, in dilute gases, line shapes are Doppler limited. Thus collisional relaxation rates may not be directly obtained from experiment but only through the deconvolution of Voigt profiles. On the other hand strong magnetic fields are required for the study of a fully resolved Doppler limited MRS. This has been previously noted for the paramagnetic gas NO [7].

In this paper we focus on the introduction in magnetic optical activity of the well known result of saturated absorption spectroscopy; that is, the elimination of the Doppler effect by subjecting the medium to 
another intense laser beam counterpropagating with respect to the probe, with a frequency close to the probe frequency. This "saturated Faraday effect " has been suggested [10] to explain modifications of the saturated Zeeman absorption spectra of $\mathrm{I}_{2}$ with magnetic field reversal [11] but, to our knowledge, no systematic study of this effect has been reported. We neglect, in this paper, the non linear effects introduced in the classical MRS by a strong magnetic field (quadratic Zeeman and Paschen Back effects [8] or by a strong probe laser [9]).

2. Doppler-free magnetic optical activity (DFMOA) - We deal with the modification of the polarization state of a tunable linearly polarized probe laser beam when it is sent through a gaseous sample. The frequency of this monochromatic probe is close to $\omega_{\mathrm{bc}}$, the frequency of an electric dipole-allowed transition $\mathrm{c} \rightarrow \mathrm{b}$ for which the Doppler width $\omega_{\mathrm{D}}$ is much larger than the Zeeman shift of levels and the collisional width.

Our calculations of electrical susceptibility have been conducted in the non linear response formalism [12] and they are similar to those used in twophoton polarization spectroscopy [13]. Density matrix elements, solutions of the Liouville equation, are calculated with the diagrammatic summation method of reference [12] in the irreducible tensor operators representation well suited in high $J$ cases [14]. Arbitrary polarization of the saturation beam has been considered but we only present here the results for the most interesting case of circularly polarization which retain the gyrotropic character of the perturbed medium (Eq. (1) is again valid here).

On the other hand, in the limit of weak strength of the saturation beam, the usual one-photon Faraday effect for Doppler broadened lines is obtained. This must be subtracted from the signal, using an appropriate modulation of the saturating beam, so that the observation is focused on the non linear part of the susceptibility which depends upon the two photons.
Our calculations of the effect have been carried out for a two-level system $J_{\mathrm{c}} \rightarrow J_{\mathrm{b}}$ to first order in probe field strength and to second order in saturating field strength.

The calculations of the effect have been carried out for a 2-level system $J_{\mathrm{c}} \rightarrow J_{\mathrm{b}}$. A «normal » Zeeman effect (triplet structure) is assumed, which is encountered in the $0 \rightarrow 1$ transition (the transition $6 s^{2}{ }^{1} S_{0} \rightarrow 6 s 6{ }^{1} S_{1}$ of $\mathrm{Ba}$, for example), or when the Landé factors of levels $b$ and $c$ are essentially the same as in the vibrational-rotational spectra of NO (X $\Pi_{1 / 2}, J>5 / 2$ ). An analytical expression has been obtained in the limit of large Doppler width. That is, integrating over the axial velocity component distribution, we assume the Doppler width to be much larger than the Zeeman splitting $H^{\prime}$ of the levels, the collisional width of the transition $\gamma_{b c}$ and the detuning $\delta_{i}=\omega_{i}-\omega_{\mathrm{bc}}$, where $i=1$, for the probe and 2 for the saturating laser frequency. The polarization of the saturating laser is taken to be right handed. Its amplitude is noted $E_{2}$ and Rabi frequency is $\beta_{2}=d E_{2} / \sqrt{2} \hbar$, where $d$ is the reduced matrix element of the electric dipole moment, $\left\langle J_{\mathrm{b}}\|d\| J_{\mathrm{c}}\right\rangle$.

We may take into account the collisional relaxation rates of the $k$ th-multipole for each level $i, \gamma_{i}^{\text {coll }}(k)$. The $\gamma_{i}^{\text {coll }}(0)$ is the rate of decay from the level $i$ through inelastic collisions; the relaxation rates of orientation $(k=1)$ and of alignment $(k=2)$ include moreover the effect of depolarizing collisions when they occur. The spontaneous emission during a collision is negligible, so the natural damping $\Gamma_{\alpha}$ is added to the collisional rate [21] :

$$
\begin{aligned}
\gamma_{\alpha}(k) & =\gamma_{\alpha}^{\mathrm{coll}}(k)+\Gamma_{\alpha} \quad \alpha=\mathrm{b}, \mathrm{c} \\
\gamma_{\mathrm{bc}} & =\gamma_{\mathrm{bc}}^{\text {coll }}(1)+\frac{1}{2}\left(\Gamma_{\mathrm{b}}+\Gamma_{\mathrm{c}}\right)
\end{aligned}
$$

where $\gamma_{b c}^{\text {coll }}(1)$ is the collisional relaxation rate of the electric dipole moment. The result is :

$$
\theta+i \Phi=C\left(\beta_{2}^{2} / \gamma_{\mathrm{bc}}\right)\left[\frac{A(J)}{x+i}-\frac{B(J)}{x+H+i}\right]
$$

Where

$$
\left.\begin{array}{c}
C=\frac{N \pi^{3 / 2} d^{2} \omega_{1} l}{3 \hbar c \omega_{\mathrm{D}}} \\
A(J)=a_{0}(J)-a_{1}(J)+a_{2}(J) \\
B(J)=a_{0}(J)+a_{1}(J)+a_{2}(J) \\
a_{0}(J)=\frac{4 J^{2} \pm 2 r(0) J}{3 J\left(4 J^{2}-1\right)}\left(\frac{1}{\gamma_{\mathrm{b}}(0)}+\frac{1}{\gamma_{c}(0)}\right) \\
a_{1}(J)=\frac{2 J^{2}-1 \mp r(1) J}{2 J\left(4 J^{2}-1\right)}\left(\frac{1}{\gamma_{\mathrm{b}}(1)}+\frac{1}{\gamma_{c}(1)}\right) \\
a_{2}(J)=\frac{2 J^{2}+3 \mp 5 r(2) J}{30 J\left(4 J^{2}-1\right)}\left(\frac{1}{\gamma_{b}(2)}+\frac{1}{\gamma_{c}(2)}\right)
\end{array}\right\} \begin{aligned}
& \text { for } \Delta J= \pm 1 \\
& \text { (R and P transitions) }
\end{aligned}
$$




$$
\left.\begin{array}{c}
a_{0}(J)=\frac{2}{3(2 J+1)}\left(\frac{1}{\gamma_{\mathrm{b}}(0)}+\frac{1}{\gamma_{\mathrm{c}}(0)}\right) \\
a_{1}(J)=\frac{1}{2 J(J+1)(2 J+1)}\left(\frac{1}{\gamma_{\mathrm{b}}(1)}+\frac{1}{\gamma_{\mathrm{c}}(1)}\right) \\
a_{2}(J)=\frac{(2 J-1)(2 J+3)}{30 J(J+1)(2 J+1)}\left(\frac{1}{\gamma_{\mathrm{b}}(2)}+\frac{1}{\gamma_{\mathrm{c}}(2)}\right)
\end{array}\right\} \begin{aligned}
& \text { for } \Delta J=0 \\
& (\mathrm{Q} \text { transition })
\end{aligned}
$$

$$
r(k)=\frac{\gamma_{b}(k)-\gamma_{c}(k)}{\gamma_{b}(k)+\gamma_{c}(k)}
$$

and where $J$ is the larger value of $J_{\mathrm{b}}$ or $J_{\mathrm{c}}$ for R and $\mathrm{P}$ transitions; $J=J_{\mathrm{b}}=J_{\mathrm{c}}$ for Q transitions. $N$ is the equilibrium population of the fundamental level c; it is assumed that the population of level $b$ is negligible.

Reduced quantities have been introduced :

$$
H=H^{\prime} / \gamma_{\mathrm{bc}}, \quad x=\delta / \gamma_{\mathrm{bc}} \text { where } \delta=\left(\delta_{1}+\delta_{2}\right) / 2 \text {. }
$$

In equation ( $4 a$ ) the upper signs hold for $\mathrm{R}$ transitions, the lower for $\mathbf{P}$ transitions.

In diatomic molecules, for example, the probability for a collision to induce transitions between rotational levels is greater than to mix the Zeeman sublevels. In such cases the depolarizing collisions may be neglected and the multipole damping rates are almost equal : $\gamma_{i}(0) \simeq \gamma_{i}(1) \simeq \gamma_{i}(2)=\gamma_{i}$. The coefficients $A(J)$ and $B(J)$ of equation (3) take the simpler form :

$$
\begin{array}{cl}
A(J)=\frac{2 J^{2} \pm 5 r J+3}{10 J\left(4 J^{2}-1\right)}\left(\frac{1}{\gamma_{\mathrm{b}}}+\frac{1}{\gamma_{\mathrm{c}}}\right) & \begin{array}{l}
\text { for } \Delta J= \pm 1 \\
(\mathrm{R} \text { and P trans }
\end{array} \\
B(J)=\frac{2\left(6 J^{2}-1\right)}{5 J\left(4 J^{2}-1\right)}\left(\frac{1}{\gamma_{\mathrm{b}}}+\frac{1}{\gamma_{\mathrm{c}}}\right) & \begin{array}{l}
\text { for } \Delta J=0 \\
(\mathrm{Q} \text { transition })
\end{array} \\
\begin{array}{c}
A(J)=\frac{(2 J-1)(2 J+3)}{10 J(J+1)(2 J+1)}\left(\frac{1}{\gamma_{\mathrm{b}}}+\frac{1}{\gamma_{\mathrm{c}}}\right) \\
B(J)=A(J)+\frac{1}{2 J(J+1)(2 J+1)}\left(\frac{1}{\gamma_{\mathrm{b}}}+\frac{1}{\gamma_{\mathrm{c}}}\right)
\end{array} & \begin{array}{l}
\gamma_{\mathrm{b}}-\gamma_{\mathrm{c}} \\
\gamma_{\mathrm{b}}+\gamma_{\mathrm{c}}
\end{array}
\end{array}
$$

Figure 1 shows the predicted Faraday rotation $\theta$ versus the reduced detuning $x$ from equation (3) and coefficients $(5 a)$ or $(5 b)$ for $\mathrm{R}, \mathrm{P}$ and $\mathrm{Q}$ transitions with $J=6$, in the extreme Doppler limit approximation, as mentioned above. Figure 2 shows $\Phi$ versus $x$ for the same transition. We notice the double absorption curve shape of $\Phi$, with one peak centred on the Zeeman resonance $\delta=-H^{\prime}$, and the other peak on the zeromagnetic field resonance (cross-over resonance). The Zeeman resonance at $\delta=+H^{\prime}$ does not appear because the saturating field is right handed polarized. The shape for $\theta$ is the corresponding dispersion profile. The widths of each of the two peaks of the $\Phi$ curves are very close to $\gamma_{b c}$, in the third order approximation.

The two peaks tend to overlap for $H^{\prime}$ values smaller and closer to $\gamma_{\mathrm{bc}}$. For well chosen $H^{\prime}$ values it may be possible to deduce the Landé factor and also $\gamma_{b c}$ from $\theta$ and $\Phi$ measurements.
There is a noticeable difference between $\mathrm{R}, \mathrm{P}$ transitions and $\mathrm{Q}$ transition behaviour, since for $\Delta J= \pm 1$, the $\theta$ and $\Phi$ curves show a strong asymmetry, and for $\Delta J=0$, both peaks have almost the same intensity. This should permit a labelling of the transitions. Another interesting feature for small values of $J(J<15$, roughly $)$ is the difference between $\Delta J=+1$ and $\Delta J=-1$ peak values of $\Phi$ at $\delta=0$ which depends through $r$ on the relative values of $\gamma_{b}$ and $\gamma_{c}$ : when $\gamma_{c} \simeq \gamma_{b}, r=0$, so the two curves are identical but when the lifetime of level $\mathrm{c}$ is much longer than the lifetime of $b, r \simeq 1$ and a large difference appears between these curves.

For the typical transition $c(J=0) \rightarrow b(J=1)$, we have carried out an analytical calculation to all orders in the saturating field strength of the part of the electrical susceptibility which does not vanish through the velocity average. For a right handed saturating field the result is :

$$
\theta+i \Phi=\frac{N \pi d^{2} \omega_{1} l i}{3 \hbar c}\left\langle\frac{1}{1-X}\left\{G_{\mathrm{b}_{,} \mathrm{c}}(1,0)\left[1+\frac{Z_{\mathrm{b}}-X_{\mathrm{b}}}{1-Y_{\mathrm{b}}}\right]-G_{\mathrm{b}_{-} \mathrm{c}}(1,0)\left[1+\frac{Z}{1-Y}\right]\right\}\right\rangle_{v}
$$




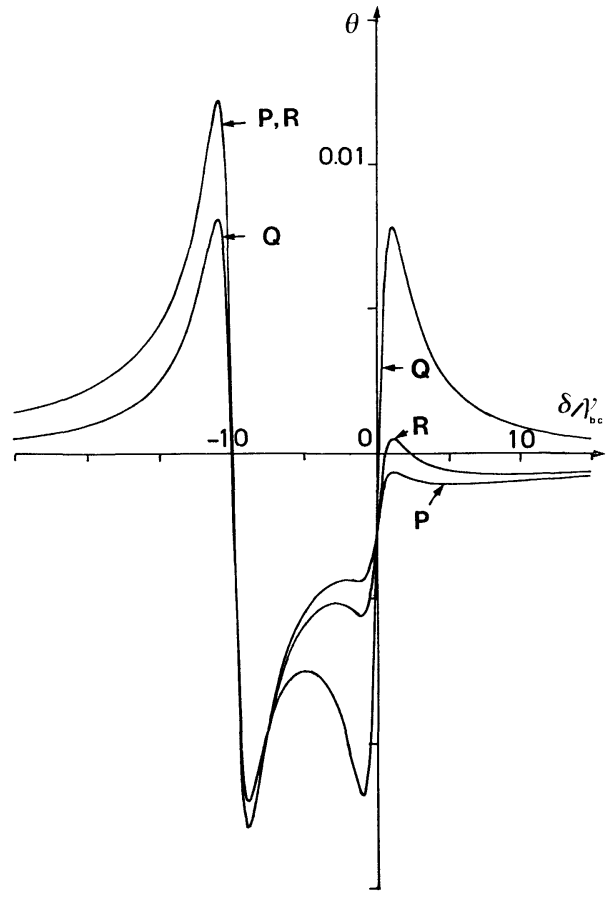

Fig. 1. - Angular rotation $\theta$ in rad. for $\mathbf{R}, \mathrm{P}$ and $\mathrm{Q}$-type transitions for $J=6, r=2 / 3, H=10, \beta_{2}=0.12 \gamma_{b c}$ as given by the third order calculation with a right handed saturating laser polarization. $C=9.65(l=1 \mathrm{~cm})$, with the conditions of reference [7] for the $\mathrm{R}(13 / 2) 1 / 2$ line of NO

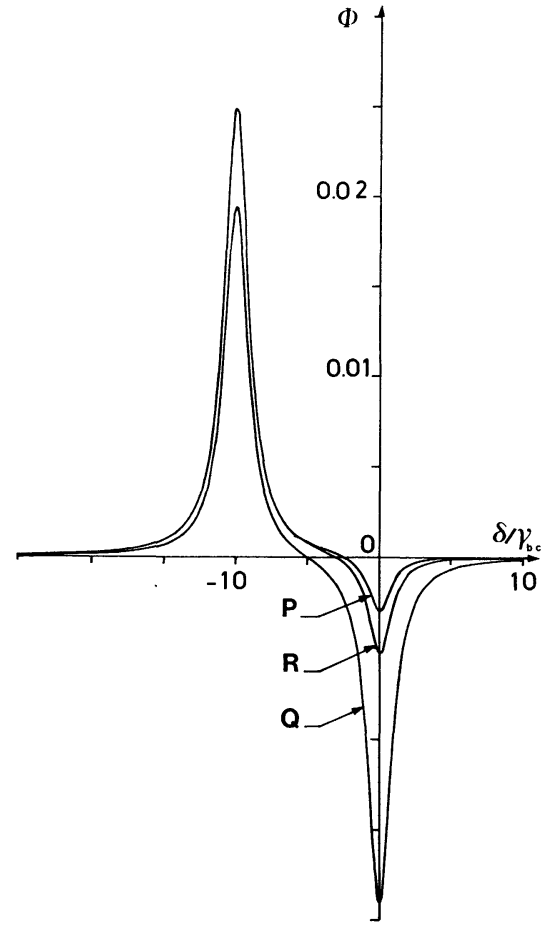

Fig. 2. - Ellipticity $\Phi$ in rad. for R, P and Q-type transitions. The values of $J, r, H, \beta_{2}, C$ and the laser polarizations are the same as in figure 1 .

where $\langle\cdots\rangle_{v}$ is the Maxwell-Boltzmann velocity average. The linear response Green functions $G_{i j}\left(n_{1}, n_{2}\right)$ is defined by :

$$
G_{i j}\left(n_{1}, n_{2}\right)=\left[n_{1}\left(\omega_{1}+\mathbf{k}_{1} \cdot \mathbf{v}\right)+n_{2}\left(\omega_{2}+\varepsilon \mathbf{k}_{2} \cdot \mathbf{v}\right)-\omega_{i j}+i \gamma_{\mathrm{bc}}\right]^{-1}
$$

with $n_{1}, n_{2}=0, \pm 1, \pm 2 ; \mathbf{k}_{1,2}$ are the wave vectors of lasers 1 and 2 which copropagate $(\varepsilon=+1)$ or counterpropagate $(\varepsilon=-1) ; i, j=\mathrm{b}_{ \pm}, \mathrm{c}$ (see Fig. 3$) ; \mathrm{b}_{ \pm}$are the sublevels $\left(m_{J}= \pm 1\right)$ of $\mathrm{b}(J=1)$

$$
\begin{aligned}
X & =\beta_{2}^{2}\left[G_{\mathrm{b}_{-} \mathrm{b}_{-}}(0,0)+G_{\mathrm{cc}}(0,0)\right]\left[G_{\mathrm{b}_{-} \mathrm{c}}(0,1)+G_{\mathrm{cb}_{-}}(0,-1)\right] \\
X_{\mathrm{b}} & =\beta_{2}^{2} G_{\mathrm{b}_{-} \mathrm{b}_{-}}(0,0)\left[G_{\mathrm{b}_{-} \mathrm{c}}(0,1)+G_{\mathrm{cb}_{-}}(0,-1)\right] \\
Y & =\beta_{2}^{2}\left[G_{\mathrm{b}_{-} \mathrm{b}_{-}}(1,-1)+G_{\mathrm{cc}}(1,-1)\right]\left[G_{\mathrm{b}_{-} \mathrm{c}}(1,0)+G_{\mathrm{cb}_{-}}(1,-2)\right] \\
Y_{\mathrm{b}} & =\beta_{2}^{2} G_{\mathrm{b}_{+} \mathrm{b}_{-}}(1,-1) G_{\mathrm{b}_{+} \mathrm{c}}(1,0) \\
Z & =\beta_{2}^{2}\left[G_{\mathrm{b}_{-} \mathrm{b}_{-}}(1,-1)+G_{\mathrm{cc}}(1,-1)\right]\left[G_{\mathrm{b}_{-} \mathrm{c}}(1,0)+G_{\mathrm{cb}_{-}}(0,-1)\right] \\
Z_{\mathrm{b}} & =\beta_{2}^{2} G_{\mathrm{b}_{+} \mathrm{b}_{-}}(1,-1)\left[G_{\mathrm{b}_{+}}(1,0)+G_{\mathrm{cb}_{-}}(0,-1)\right] .
\end{aligned}
$$

Figure 3 shows the coupling of the sublevels by the two lasers with their polarizations indicated.

Figures 4 and 5 show $\theta$ and $\Phi$ versus $\delta / \gamma_{\mathrm{bc}}$ in the case of identical detunings for the two lasers $\delta_{1}=\delta_{2}$ and for different values of the Rabi frequency $\beta_{2}$.

For values of $\beta_{2}<\gamma_{\mathrm{bc}}$ we obtain the shapes given in figures 1 and 2 for $\Delta J=+1(J=1)$, from the third order calculation. With increasing values of $\beta_{2}$, the signals become more and more broadened, due to power broadening. The resonance peaks are deformed and shifted by this broadening. This behaviour suggests that for experiments a ratio $\beta_{2} / \gamma_{b c}<1$, should be taken in order to keep the simpler interpretation

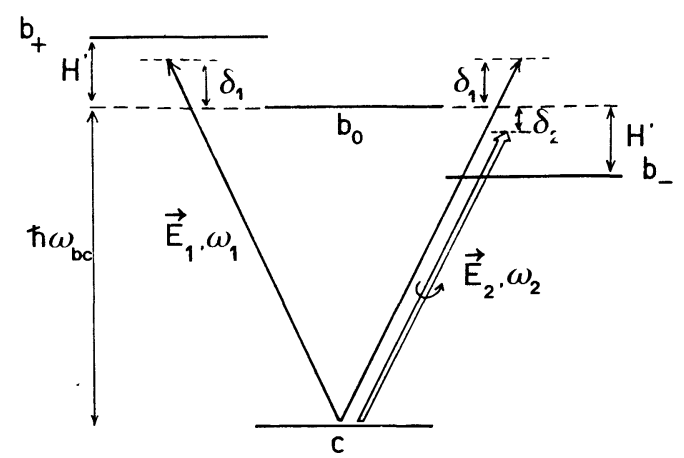

Fig. 3. - Scheme of the two-level system $(0 \rightarrow 1)$ and laser polarizations. 


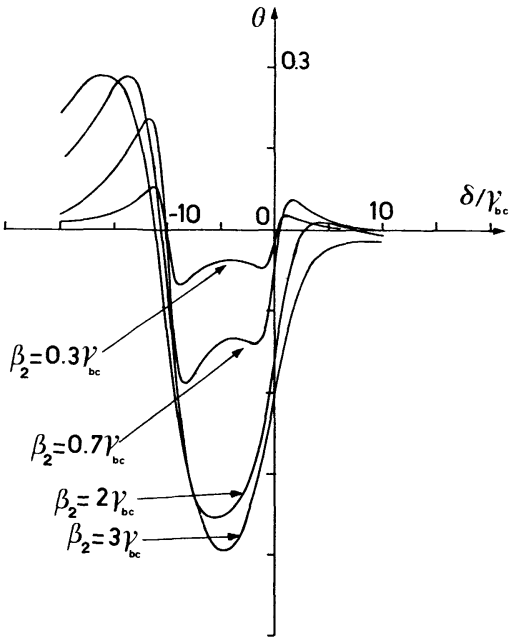

Fig. 4. - Angular rotation $\theta$ in arbitrary units for the $0 \rightarrow 1$ transition, at $H=10, r=0$ for different values of $\beta_{2} / \gamma_{\mathrm{bc}}(0.3$, $0.7,2,3)$

of $\theta$ and $\Phi$ measurements in terms of $\gamma_{b}, \gamma_{c}, \gamma_{b c}$ and Zeeman splitting as given by equation (3) with the coefficients $(5 a),(5 b),(5 c)$.

3. Discussion and conclusion. - The zero-magnetic field limits of expressions (3) lead to the Doppler-free polarization spectroscopy (DFPS) ; they are consistent with the expressions of Wieman and Hänsch [15]. A different $J$-dependence for $Q$ and R-P transitions was noted for DFPS [16]; this remark applies also to DFMOA and should help, as mentioned, for labelling of molecular lines ; taking advantage that the $Q$ transition signals in DFMOA may be more intense than the corresponding $\mathrm{Q}$ branch of DFPS.

An all-order calculation of DFPS signals has been computed by Ritze et al. [17] for high $J$ values. Such a sum on all magnetic sublevels of terms similar to the $0 \rightarrow 1$ expressions (6) may be carried out numerically

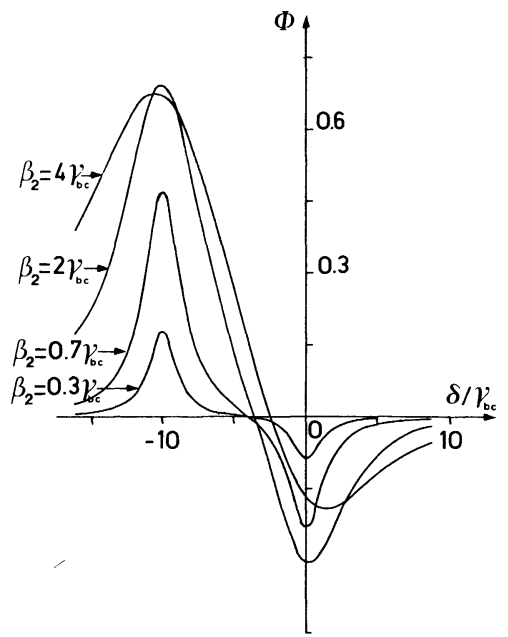

Fig. 5. - Ellipticity $\Phi$ in arbitrary units for the $0 \rightarrow 1$ transition at $H=10, r=0$ for different values of $\beta_{2} / \gamma_{\mathrm{bc}}(0.3,0.7,2,4) . C=9.65$ $(l=1 \mathrm{~cm})$.

for DFMOA signals. It is beyond the scope of this paper since we have seen, for the $0 \rightarrow 1$ transition, that no further significant information can be obtained from very strong saturating fields experiments.

With $\theta$ and $\Phi$ (or MRS) measurements, it is possible to extract the collisional relaxation rate of the transition, from widths of the observed peaks. The magnetic fields involved in DFMOA are much weaker than in the usual MOA experiments, so the relaxation rates should be unaffected by them [20]. Even very small magnetic moments may be measured from the magnetic splitting.

Saturated absorption techniques with magnetic field also have been introduced by some authors through Zeeman absorption spectra in $\mathrm{CH}_{4}$ [18], or the Hanle effect in absorption [19]. The signal-tonoise ratio of the DFMOA should be better than in saturated absorption measurements.

\section{References}

[1] Buckingham, A. D. and Stephens, P. J., Ann. Rev. Phys. Chem. 17 (1966) 399.

In some cases, for instance $\mathrm{O}_{2}$, the magnetical susceptibility $\chi^{(\mathrm{m})}$ has to be taken into account so equation (1) becomes :

$$
\theta+i \Phi=\frac{2 \pi \omega l}{i c}\left[\chi_{x y}^{(\mathrm{e})}+\chi_{x y}^{(\mathrm{m})}\right],
$$

as shown by Hougen, J. T., J. Chem. Phys. 32 (1960) 1122.

[2] Reference [1] and a few other review articles:

SChatz, P. N. and MC CAFFerTy, A. J., Quart. Rev. 23 (1970) 552 ;

Caldwell, D., Thorne, J. M. and Eyring, H., Ann. Rev. Phys. Chem. 22 (1971) 259;

BRIAT, B., Monographie, October 1973, p. 375.

[3] Carroll, T., Phys. Rev. 52 (1937) 822.

[4] This technique of vibrational analysis based on the theory of reference [3], was proposed and used for alkali metal molecules by Loomis, F. W. and Nusbaum, R. E., Phys.
Rev. 38 (1931) 1447 and has been reintroduced many years later by Eberhard, W. H., Wu Chieh Cheng and Renner, H., J. Mol. Spectrosc. 3 (1959) 664 for $\mathrm{A}^{3} \Pi_{1} \rightarrow \mathrm{X}^{1} \Sigma$ systems of $\mathrm{ICl}$ and $\mathrm{IBr}$, and by KusCH, $\mathrm{P}$. and Hessel, M. M., J. Mol. Spectrosc. 32 (1969) 181 for the very dense ${ }^{1} \Pi_{\mathrm{u}} \rightarrow \mathrm{X}^{1} \Sigma^{+}$system of $\mathrm{Cs}_{2}$.

[5] Moe, G. and Happer, W., Proc. Second Int. Conf. on Spectral Lines, Eugene, Oregon (1974).

[6] Roberts, G. J., Baird, P. E. G., Brimicombe, M. W. S. M., Sandars, P. G. M., Selby, D. R. and Stacey, D. N., $J$. Phys. B 13 (1980) 1389.

[7] Blum, F. A., Nill, K. W. and Strauss, A. J., J. Chem. Phys. 58(1973) 4968.

[8] Manakov, N. L., Ovsiannikov, V. D. and Kielich, S., Phys. Rev. A, to be published.

[9] Kielich, S., Manakov, N. L. and Ovsiannikov, V. D., Acta Phys. Pol. A 53 (1978) 737 ;

Yu, Y. J. and Osborn, R. K., Phys. Rev. A 15 (1977) 2404. [10] VIGUE, J., Thesis, Université de Paris-VI (1978), unpublished. 
[11] Sorem, M. S., Microwave Laboratory Report 2082 (1972), Stanford University.

[12] Ben Reuven, A. and Klein, L., Phys. Rev. A 4 (1971) 753 ; Klein, L., Giraud, M. and Ben Reuven, A., Phys. Rev. A 10 (1974) 682.

[13] Kaftandian, V. P., Klein, L. and Talin, B., J. Physique 40 (1979) 1037.

[14] Ben Reuven, A., Phys. Rev. 141 (1966) 34 ; Phys. Rev. 145 (1966) 7. At very high $J$, the Ducloy formalism should be better :

Ducloy, M., J. Physique 36 (1975) 927.

[15] Wieman, C. and HänsCh, T., Phys. Rev. Lett. 36 (1976) 1170.

[16] SaÏkan, S., J. Opt. Soc. Am. 68 (1978) 1184 ;
Teets, R., Feinberg, R., Hänsch, T. W. and SChawlow, A. L., Phys. Rev. Lett. 37 (1976) 683 ;

Stert, V. and Fischer, R., Appl. Phys. 17 (1978) 151.

[17] Ritze, H. H., Stert, V. and Meisel, E., Opt. Commun. 29 (1979) 51.

[18] Uzgiris, E. E., Hall, J. L. and Barger, R. L., Phys. Rev. Lett. 26 (1971) 289.

[19] Colomb, I., Gorlicki, M. and Dumont, M., Opt. Commun. 21 (1977) 289.

[20] Омоnт, A., J. Physique 34 (1973) 179.

[21] Omont, A., «Irreducible Components of the Density Matrix. Application to Optical Pumping " in Prog. Quantum Electronics 5 (1977) 69. 